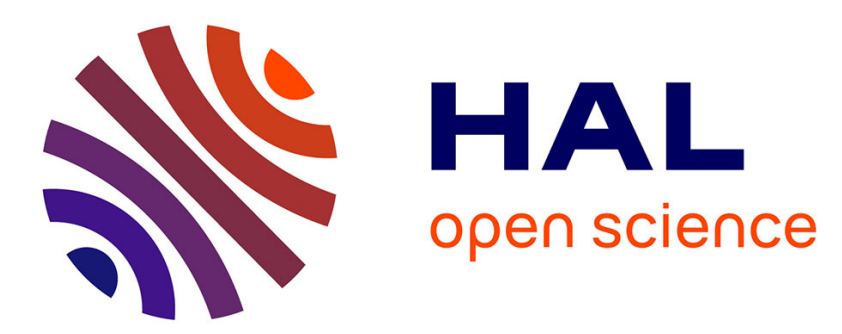

\title{
Simulation numérique globale de la décharge impulsionnelle d'une génératrice asynchrone sans fer
}

K. Ly, G. Quichaud, C. Rioux, F. Sultanem

\section{To cite this version:}

K. Ly, G. Quichaud, C. Rioux, F. Sultanem. Simulation numérique globale de la décharge impulsionnelle d'une génératrice asynchrone sans fer. Revue de Physique Appliquée, 1980, 15 (8), pp.1375-1382. 10.1051/rphysap:019800015080137500 . jpa-00244861

\section{HAL Id: jpa-00244861 https://hal.science/jpa-00244861}

Submitted on 1 Jan 1980

HAL is a multi-disciplinary open access archive for the deposit and dissemination of scientific research documents, whether they are published or not. The documents may come from teaching and research institutions in France or abroad, or from public or private research centers.
L'archive ouverte pluridisciplinaire HAL, est destinée au dépôt et à la diffusion de documents scientifiques de niveau recherche, publiés ou non, émanant des établissements d'enseignement et de recherche français ou étrangers, des laboratoires publics ou privés. 


\title{
Simulation numérique globale de la décharge impulsionnelle d'une génératrice asynchrone sans fer
}

\author{
K. Ly, G. Quichaud, C. Rioux et F. Sultanem \\ Laboratoire d'Electrotechnique des Universités Paris VI et XI, Bât. 214, 91405 Orsay, France
}

(Reçu le 11 mars 1980, accepté le 28 avril 1980)

\begin{abstract}
Résumé. - Les auteurs effectuent une analyse numérique d'une source d'énergie électrique impulsionnelle constituée par une génératrice asynchrone sans fer à rotor discoïde massif et à charge accordée (capacité de compensation de facteur de puissance, pont redresseur, inductance de stockage). Le programme de simulation automatique de convertisseurs statiques sur ordinateur (SACSO) a été modifié à cet effet par l'introduction de couplages résistifs. Les résultats ainsi obtenus pour une décharge complète ( 200 périodes) au moyen de deux modélisations de la machine asynchrone sont en accord avec l'expérience, les écarts maximums étant inférieurs à $20 \%$.
\end{abstract}

\begin{abstract}
The authors have done a numerical analysis of a high pulse energy generation system which consists of an asynchronous, iron-free solid rotor generator with a resonating load (power factor correction capacitors plus a full wave diode bridge and an inductive load). The automatic simulation program for the analysis of the static converters (SACSO) was modified by the introduction of resistive coupling terms in the network matrices. Results have been obtained for a complete discharge ( 200 periods) using two distinct models of the machine and are accurate to within $20 \%$ of the practical system tested.
\end{abstract}

1. Introduction. - La réalisation de sources d'énergie électrique impulsionnelles puissantes à faible coût présente un intérêt certain pour diverses applications telles que le formage magnétique, l'alimentation de souffleries à rafales, les expériences de fusion contrôlée, etc...

Parmi toutes les solutions, une des moins onéreuses consiste à stocker l'énergie dans un volant en rotation couplé à une génératrice $[1,2]$. Dans un premier temps, le système volant-génératrice est couplé à une bobine magnétique sans fer; la génératrice transfère rapidement la plus grande partie de l'énergie du volant sous forme magnétique dans la bobine. Dans un deuxième temps, le courant de la bobine serait dérivé vers l'utilisation à l'aide d'un disjoncteur spécial.

Pour que le premier transfert d'énergie soit acceptable il faut qu'il soit nettement plus court que la constante de temps de la bobine, qui est au plus de quelques secondes lorsque la masse totale de cuivre est de l'ordre de la tonne. Par suite la génératrice doit être très puissante : il a été choisi pour cela une génératrice asynchrone sans fer.

Le dispositif volant-génératrice-bobine est alors associé à un système de condensateurs permettant de fournir à chaque instant la puissance réactive nécessaire, tandis que le courant alternatif est trans- formé en continu par un pont redresseur avant d'être envoyé dans la bobine de stockage.

$\mathrm{Si}$ un tel dispositif permet de résoudre le problème global, qui est le transfert rapide avec un bon rendement de l'énergie du volant dans la bobine il présente aussi un certain nombre d'inconnues. En particulier si la structure sans fer permet des puissances très élevées [3] grâce aux hautes inductions, il en résulte un accroissement important des inductances de fuites. Les conditions de couplage du système résonnant machine-condensateur s'en trouvent également perturbées d'une manière qu'il est difficile d'analyser par un raisonnement simple.

L'étude rapportée ici a pour but d'effectuer une modélisation numérique complète de fonctionnement $\mathrm{du}$ système volant-génératrice-condensateur pont redresseur-bobine de stockage en nous bornant à une machine asynchrone sans fer à rotor massif simplifiée.

2. Description d'une décharge impulsionnelle. La figure 1 donne le schéma du dispositif à simuler $[4,5]$. La génératrice asynchrone sans fer est à rotor discoïde [2]. Le rotor monobloc (Fig. 2) en alliage d'aluminium AU4G de $1 \mathrm{~m}$ de diamètre et $5 \mathrm{~cm}$ d'épaisseur est placé entre deux bobinages plats (Fig. 3) à $2 q=8$ pôles. Son inertie $J=13,5 \mathrm{~kg} \cdot \mathrm{m}^{2}$ 


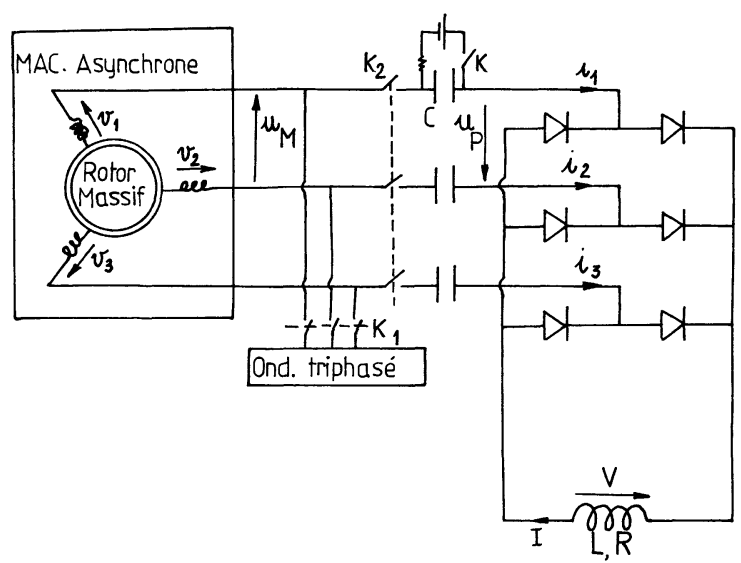

Fig. 1. - Schéma de l'ensemble à simuler.

[Simulated system.]

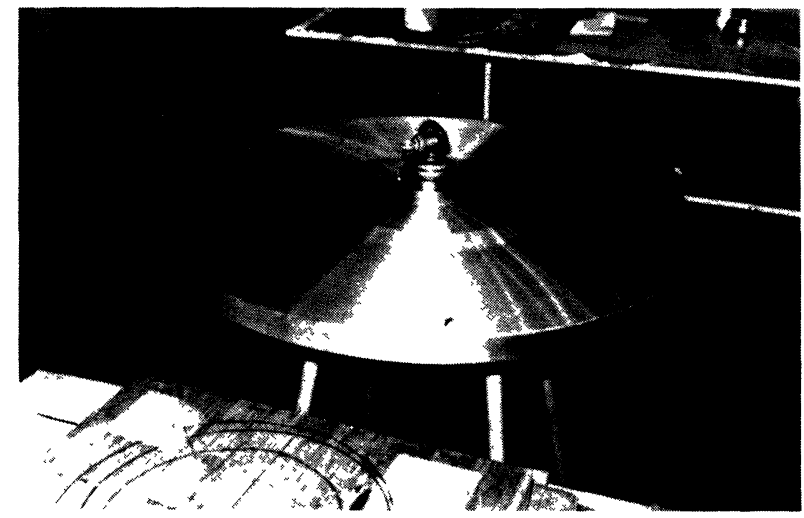

Fig. 2. - Vue du rotor.

[Rotor view.]

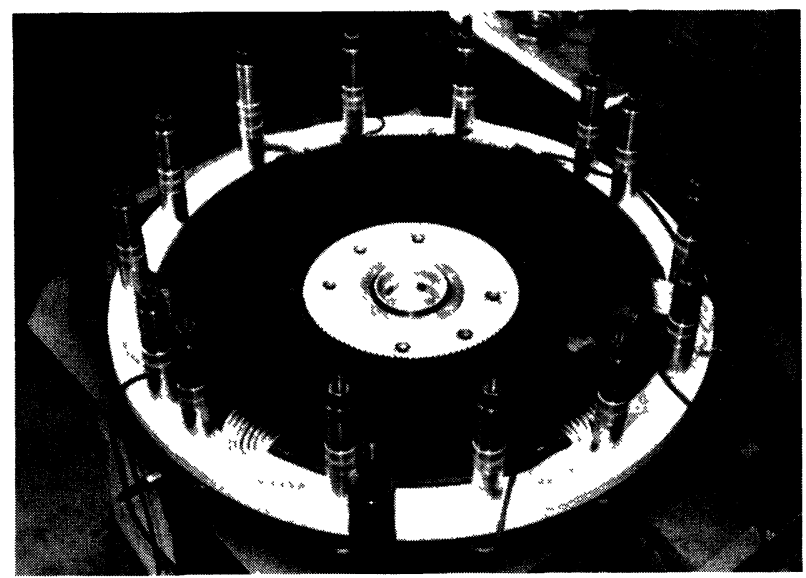

Fig. 3. - Vue des bobinages d'un des stators.

[Winding view of one stator.]

conduit à une énergie stockée voisine du mégajoule à $3600 \mathrm{t} / \mathrm{min}$. Le but de l'expérience est de transférer cette énergie dans une inductance de charge d'inductance $L=0,25 \mathrm{mH}$ et de résistance $R=0,12 \Omega$, d'où ultérieurement grâce à des disjoncteurs rapides, elle pourra être utilisée comme source d'énergie secondaire.

En nous reportant à la figure 1, décrivons brièvement une expérience de décharge. On lance tout d'abord le rotor $\left(\mathrm{K}_{2}\right.$ ouvert, $\mathrm{K}_{1}$ fermé) à une vitesse initiale $N_{0}=3600 \mathrm{t} / \mathrm{min}$. grâce à un onduleur triphasé. Après la précharge d'un condensateur $C=1000 \mu \mathrm{F}$ (K fermé) à la tension $V_{\mathrm{c}_{0}}=1000 \mathrm{~V}$, la décharge complète se déroule de la façon suivante, comme on peut le voir sur les oscillogrammes des figures 4 et 5 après ouverture de $\mathrm{K}_{1}$, $\mathrm{K}$ et fermeture de $\mathrm{K}_{2}$ à l'instant $t=0$.

Une première phase d'une durée de quelques millisecondes correspond à la décharge préliminaire, dans l'inductance de charge $L$ et dans la machine, du condensateur préchargé.

Une deuxième phase d'une durée de quelques dizaines de millisecondes correspond à ce que nous conviendrons d'appeler l'amorçage interne de la génératrice, le pont de Graetz étant en court-circuit.

La troisième phase d'une durée de quelques dizièmes de seconde correspond à la décharge utile pendant laquelle une fraction de l'énergie du rotor est transférée dans l'inductance de charge $L$.

Enfin la dernière phase d'une durée également de quelques dizièmes de seconde correspond au désamorçage interne de la génératrice asynchrone, le pont de Graetz étant à nouveau en court-circuit.

Si lors de l'amorçage ou du désamorçage internes les tensions et courants côté machine ont des allures proches de sinusoïdes amplifiées ou amorties [5] il n'en est plus de même lors de la décharge utile comme le montrent les oscillogrammes de la figure 6 pour lesquels le courant machine est trapézoïdal et la tension composée à l'entrée du pont est différente d'une sinusoïde.

3. Principe de la simulation globale. - La simulation numérique globale effectuée repose sur l'utili-

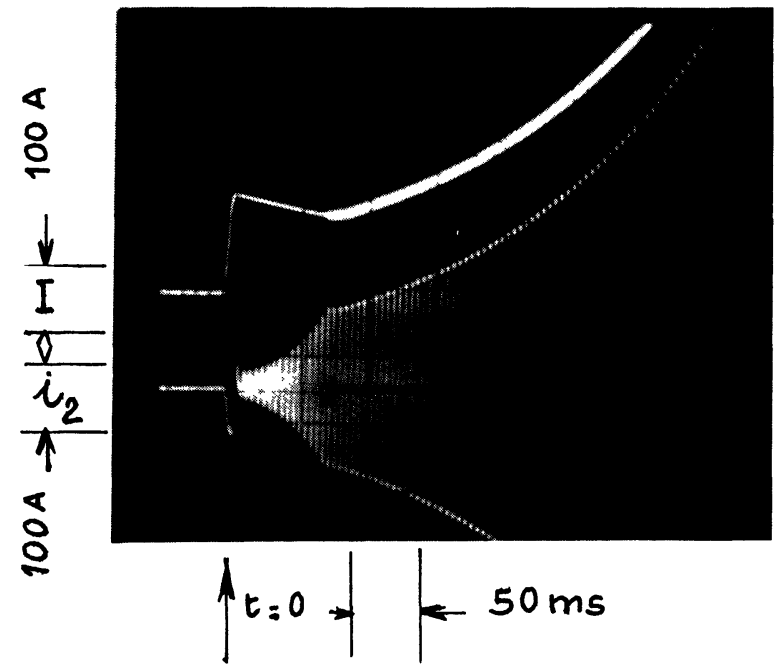

Fı̀. 4. - Óscillogrammes montrant le début de la décharge.

[Oscillograms of the beginning of the discharge.] 


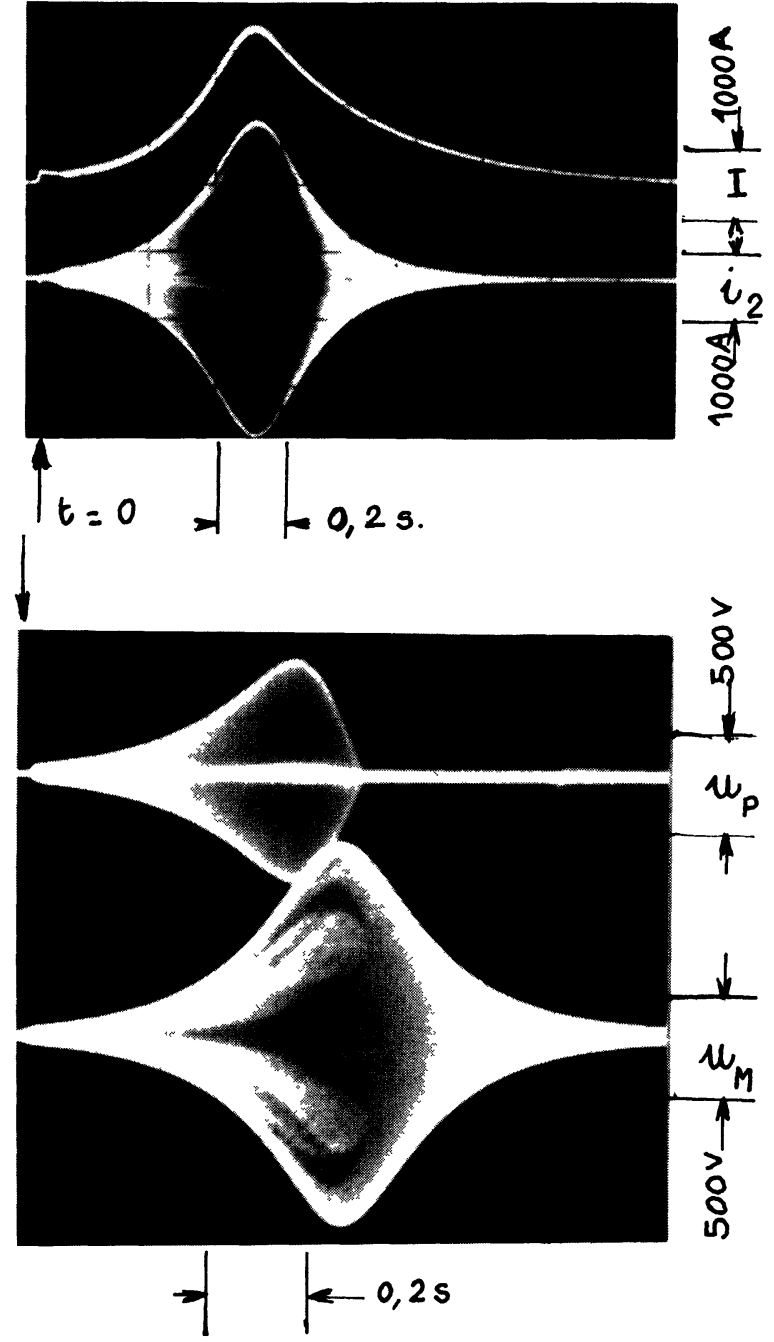

Fig. 5. - Evolution des courants et tensions lors d'une décharge complète.

[Currents and voltages evolution during total discharge.]

sation du programme de simulation automatique de convertisseurs statiques sur ordinateur (SACSO) mis au point au Laboratoire d'Electrotechnique et d'Electronique industrielle de l'ENSEEIH de Toulouse $[6,7]$. Ce programme est destiné à simuler les circuits statiques de l'électronique de puissance comprenant des résistances, capacités, inductances, sources de tensions et soupapes. Ces dernières, diodes ou thyristors sont représentées par des résistances à deux états, l'une $R_{\infty}$ de forte valeur correspondant à l'état bloqué, l'autre $R_{0}$ de faible valeur correspondant à l'état passant. Ainsi entre deux configurations de
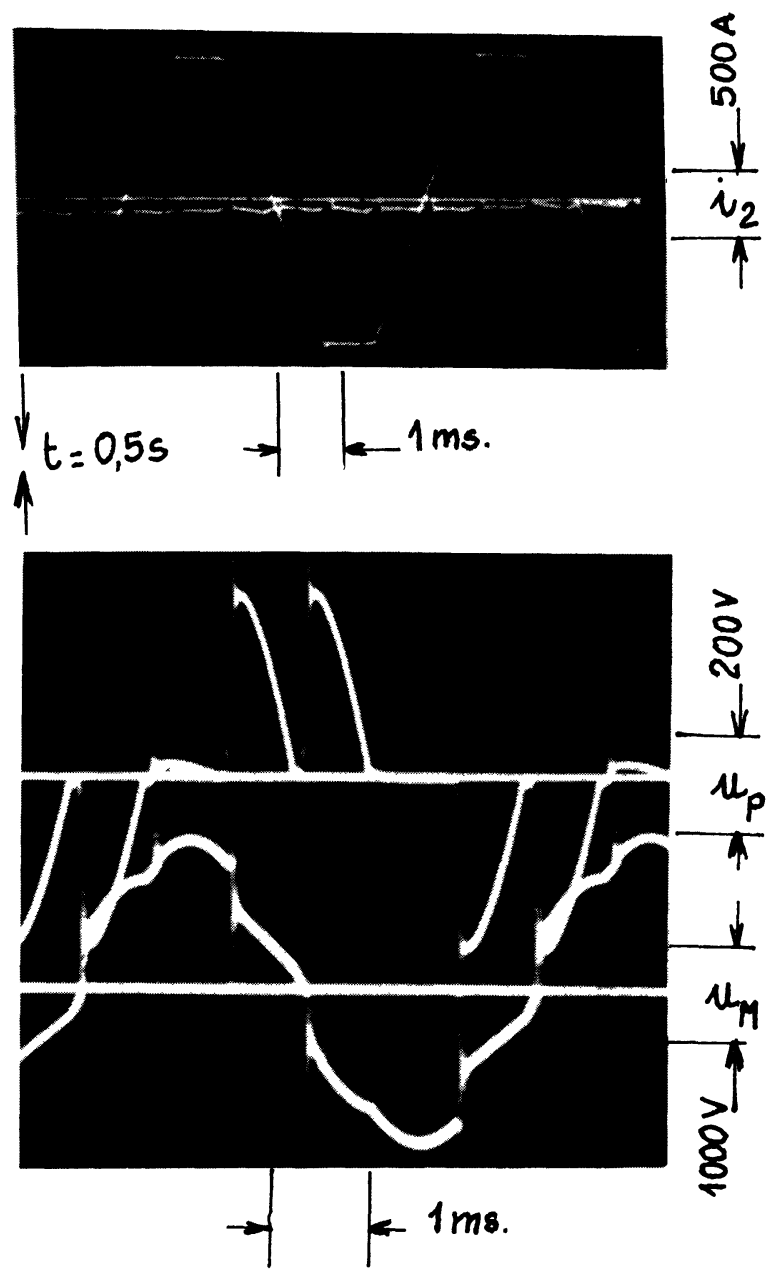

Fig. 6. - Formes des tensions et courants lors de la décharge utile.

[Currents and voltages waveforms during useful discharge.]

soupapes l'évolution du réseau est déterminée par l'intégration de son équation d'état au moyen du calcul d'une exponentielle de matrice. Ce programme est donc bien adapté pour traiter le pont de Graetz et la charge inductive. Mais nous avons dû cependant le modifier [5] en ajoutant les éléments couplages résistifs afin qu'il accepte aussi un modèle statique de machine asynchrone dans lequel il apparaît des f.e.m. dynamiques proportionnelles à des courants.

4. Modèle de machine asynchrone. - 4.1 MoDÈLE DIPHASÉ ET TRIPHASÉ. - Le modèle le plus simple est un modèle diphasé classique satisfaisant aux équations suivantes où $\Omega$ est la pulsation de rotation du rotor :

$$
\begin{aligned}
& -v_{\mathrm{d}}=\frac{2}{3} R_{\mathrm{s}} i_{\mathrm{d}}+\frac{2}{3} L_{\mathrm{s}} \frac{\mathrm{d} i_{\mathrm{d}}}{\mathrm{d} t}+\frac{2}{3} M \frac{\mathrm{d} j_{\mathrm{d}}}{\mathrm{d} t}, \\
& -v_{\mathrm{q}}=\frac{2}{3} R_{\mathrm{s}} i_{\mathrm{q}}+\frac{2}{3} L_{\mathrm{s}} \frac{\mathrm{d} i_{\mathrm{q}}}{\mathrm{d} t}+\frac{2}{3} M \frac{\mathrm{d} j_{\mathrm{q}}}{\mathrm{d} t}, \\
& -v_{\mathrm{h}}=\frac{2}{3} R_{\mathrm{s}} i_{\mathrm{h}},
\end{aligned}
$$




$$
\begin{aligned}
& 0=\frac{2}{3} R_{\mathrm{r}} j_{\mathrm{d}}+\frac{2}{3} L_{\mathrm{r}}\left(\frac{\mathrm{d} j_{\mathrm{d}}}{\mathrm{d} t}-q \Omega j_{\mathrm{q}}\right)+\frac{2}{3} M\left(\frac{\mathrm{d} i_{\mathrm{d}}}{\mathrm{d} t}-q \Omega i_{\mathrm{q}}\right), \\
& 0=\frac{2}{3} R_{\mathrm{r}} j_{\mathrm{q}}+\frac{2}{3} L_{\mathrm{r}}\left(\frac{\mathrm{d} j_{\mathrm{q}}}{\mathrm{d} t}+q \Omega j_{\mathrm{d}}\right)+\frac{2}{3} M\left(\frac{\mathrm{d} i_{\mathrm{q}}}{\mathrm{d} t}+q \Omega i_{\mathrm{d}}\right),
\end{aligned}
$$

où $v_{\mathrm{d}}, v_{\mathrm{q}}, v_{\mathrm{h}}$ et $i_{\mathrm{d}}, i_{\mathrm{q}}, i_{\mathrm{h}}$ sont définis à partir des tensions $v_{1}, v_{2}, v_{3}$ et des courants $i_{1}, i_{2}, i_{3}$ des phases du stator de la machine :

$$
\begin{array}{ll}
v_{\mathrm{d}}=\frac{2}{3}\left(v_{1}-\frac{v_{2}}{2}-\frac{v_{3}}{2}\right) & i_{\mathrm{d}}=i_{1}-\frac{i_{2}}{2}-\frac{i_{3}}{2}, \\
v_{\mathrm{q}}=\frac{2}{3}\left(-\frac{\sqrt{3}}{2} v_{2}+\frac{\sqrt{3}}{2} v_{3}\right) & i_{\mathrm{q}}=-\frac{\sqrt{3}}{2} i_{2}+\frac{\sqrt{3}}{2} i_{3}, \\
v_{\mathrm{h}}=\frac{2}{3}\left(\frac{v_{1}}{2}+\frac{v_{2}}{2}+\frac{v_{3}}{2}\right) & i_{\mathrm{h}}=\frac{i_{1}}{2}+\frac{i_{2}}{2}+\frac{i_{3}}{2} .
\end{array}
$$

Quant au couple développé sur l'arbre il se met sous la forme suivante :

$$
\Gamma=\frac{2}{3} q M\left(i_{\mathrm{d}} j_{\mathrm{q}}-i_{\mathrm{q}} j_{\mathrm{d}}\right) .
$$

Afin d'éviter (comme nous le verrons dans la suite lors de la simulation) l'utilisation d'un transformateur Scott nous avons montré [5] l'équivalence du modèle précédent au modèle triphasé suivant :

$$
\begin{aligned}
-\left[V_{\mathrm{T}}\right] & =R_{\mathrm{s}}\left[I_{\mathrm{T}}\right]+\frac{2}{3} L_{\mathrm{s}}[A] \frac{\mathrm{d}}{\mathrm{d} t}\left[I_{\mathrm{T}}\right]+\frac{2}{3} M[A] \frac{\mathrm{d}}{\mathrm{d} t}\left[J_{\mathrm{T}}\right], \\
0 & =R_{\mathrm{r}}\left[J_{\mathrm{T}}\right]+L_{\mathrm{r}} \frac{\mathrm{d}\left[J_{\mathrm{T}}\right]}{\mathrm{d} t}+\frac{2}{3} M[A] \frac{\mathrm{d}}{\mathrm{d} t}\left[I_{\mathrm{T}}\right]-\left[E_{\mathrm{T}}\right], \\
{\left[E_{\mathrm{T}}\right] } & =q \Omega[B]\left(M\left[I_{\mathrm{T}}\right]-L_{\mathrm{r}}\left[J_{\mathrm{T}}\right]\right),
\end{aligned}
$$

ou

$$
[A]=\left[\begin{array}{ccc}
1 & -1 / 2 & -1 / 2 \\
-1 / 2 & 1 & -1 / 2 \\
-1 / 2 & -1 / 2 & 1
\end{array}\right] \quad \text { et } \quad[B]=\frac{\sqrt{3}}{3}\left[\begin{array}{rrr}
0 & -1 & 1 \\
1 & 0 & -1 \\
-1 & 1 & 0
\end{array}\right]
$$

$\left[V_{\mathrm{T}}\right]$ et $\left[I_{\mathrm{T}}\right]$ sont respectivement les matrices colonne des tensions $v_{1}, v_{2}, v_{3}$ et courants $i_{1}, i_{2}, i_{3}$ alors que $\left[J_{\mathrm{T}}\right]$ est la matrice colonne de trois courants $j_{1}, j_{2}, j_{3}$ équivalents aux courants induits dans le rotor. Par ailleurs dans ces conditions le couple a pour expression :

$$
\Gamma=\frac{\sqrt{3}}{3} q M\left(j_{1}\left(i_{2}-i_{3}\right)+j_{2}\left(i_{3}-i_{1}\right)+j_{3}\left(i_{1}-i_{2}\right)\right) .
$$

Notons que dans le cas particulier d'un montage étoile des phases sans fil neutre les deux premières équations du modèle triphasé se simplifient sous la forme suivante :

$$
\begin{aligned}
-\left[V_{\mathrm{T}}\right] & =R_{\mathrm{s}}\left[I_{\mathrm{T}}\right]+L_{\mathrm{s}} \frac{\mathrm{d}}{\mathrm{d} t}\left[I_{\mathrm{T}}\right]+M \frac{\mathrm{d}}{\mathrm{d} t}\left[J_{\mathrm{T}}\right], \\
0 & =R_{\mathrm{r}}\left[J_{\mathrm{T}}\right]+L_{\mathrm{r}} \frac{\mathrm{d}}{\mathrm{d} t}\left[J_{\mathrm{T}}\right]+M \frac{\mathrm{d}}{\mathrm{d} t}\left[I_{\mathrm{T}}\right]-\left[E_{\mathrm{T}}\right],
\end{aligned}
$$

4.2 DÉTERMINATION DES PARAMÈTRES DES MODÈLES. - Pour les simulations numériques de la machine réelle le choix des paramètres des modèles diphasé ou triphasé a été effectué en approximant au mieux l'impédance cyclique $Z_{\Omega}(\omega)$ de la machine réelle (où $\omega$ est la pulsation d'alimentation de la machine).
Les modèles précédents conduisent aux expressions suivantes :

$$
z(\omega-q \Omega)=\frac{Z_{\Omega}(\omega)-R_{\mathrm{s}}}{\omega}=G(\omega-q \Omega)+j H(\omega-q \Omega),
$$


où :

$$
\begin{aligned}
& G(\omega-q \Omega)=\frac{R_{\mathrm{r}} M^{2} g \omega}{R_{\mathrm{r}}^{2}+L_{\mathrm{r}}^{2} g^{2} \omega^{2}} \\
& H(\omega-q \Omega)=L_{\mathrm{s}}-\frac{M^{2} L_{\mathrm{r}} g^{2} \omega^{2}}{R_{\mathrm{r}}^{2}+L_{\mathrm{r}}^{2} g^{2} \omega^{2}} .
\end{aligned}
$$

Sur la figure 7 nous avons porté la courbe de description de $z$ en fonction de la pulsation de glissement $g \omega=\omega-q \Omega$ ainsi que la courbe correspondant à nos modèles approchés lorsque nous prenons :

$$
\begin{aligned}
& L_{\mathrm{s}}=0,754 \mathrm{mH} \\
& R_{\mathrm{r}}=0,022 \Omega \\
& L_{\mathrm{r}}=0,659 \mathrm{mH} \\
& M=0,600 \mathrm{mH} \\
& R_{\mathrm{s}}=0,15 \Omega .
\end{aligned}
$$

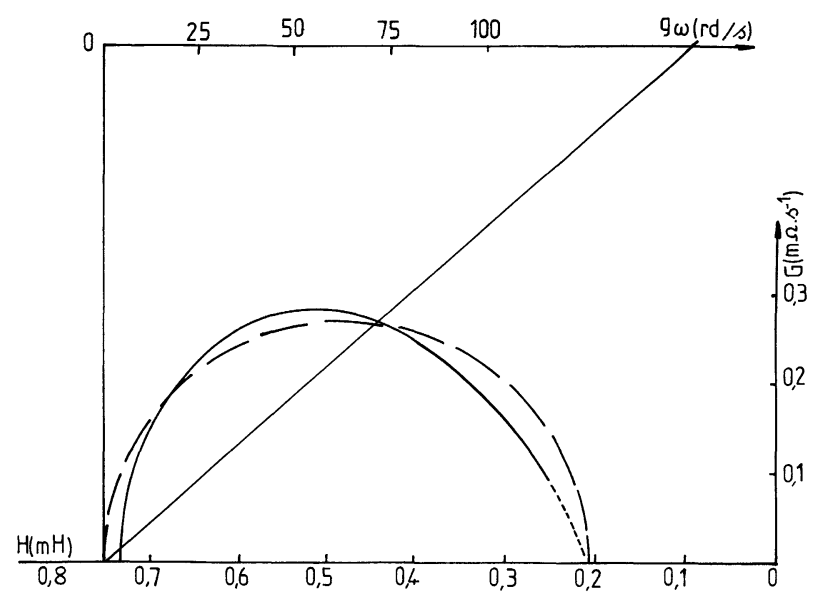

Fig. 7. - Courbes de description d'impédance de la machine : - courbe expérimentale ; --- courbe approchée utilisée lors de la simulation.

[Diagram of machine impedance : - experimental curve; --- approximative curve used in simulation.]

5. Mise en euvre et résultats de la simulation. Les figures 8 et 9 donnent les deux circuits équivalents dont la simulation a été effectuée [5] au moyen du programme SACSO modifié. Dans l'un, on a utilisé le modèle diphasé défini précédemment ; ceci nécessite un transformateur Scott d'adaptation dont les valeurs des éléments sont données sur la figure 8 . Pour le second circuit avec modèle triphasé (Fig. 9) ce transformateur est inutile. Dans les deux cas les résistances à l'état bloqué et à l'état passant des diodes ont été prises respectivement égales à $R_{\infty}=500 \Omega$ et $R_{0}=0,001 \Omega$, l'inductance de charge ayant les caractéristiques données lors de la description de l'expérience.

Nous donnons dans la suite les résultats d'une simulation effectuée dans les conditions des relevés oscillographiques des figures 4 à 6 . Chaque condensateur de compensation de puissance réactive a une valeur $C=1000 \mu \mathrm{F}$ et l'un d'eux est préchargé à une

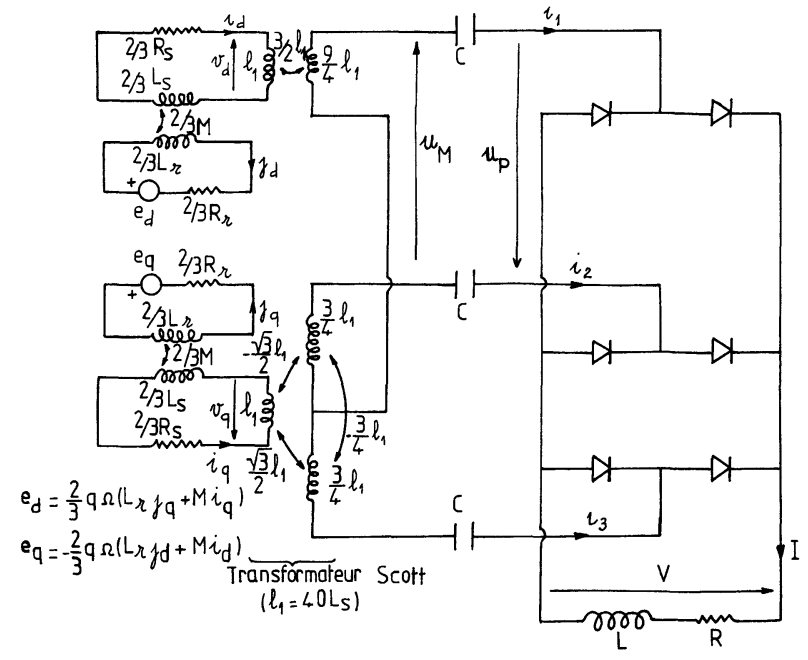

Fig. 8. - Modèle diphasé complet.

[Complete two-phase model.]

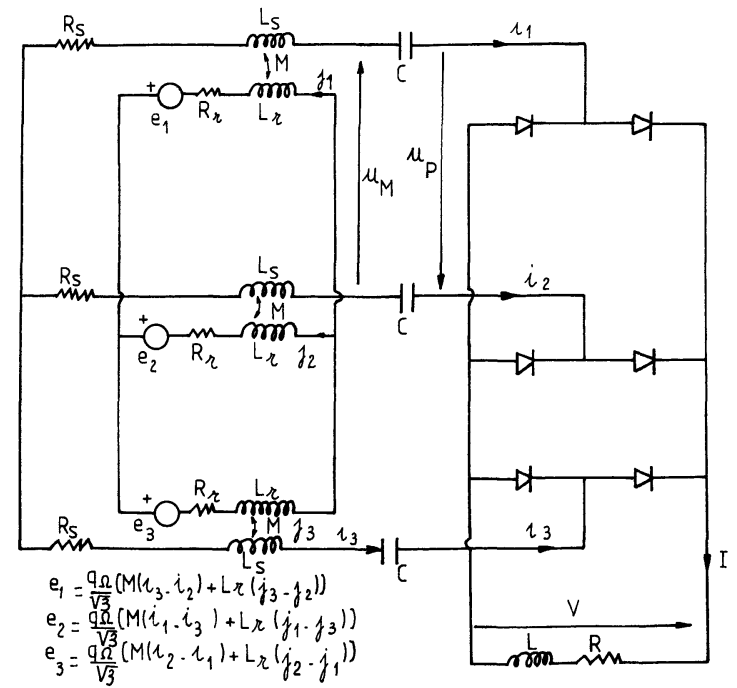

Fig. 9. - Modèle triphasé complet.

[Complete three-phase model.]

tension $V_{\mathrm{c}_{0}}=1000 \mathrm{~V}$, la vitesse initiale du rotor étant $N_{0}=3600 \mathrm{t} / \mathrm{min}$. à l'instant $t=0$.

Notons que l'absence de sources permet de conserver lors de la simulation les condensateurs en étoile si on a pris soin d'éliminer du programme SACSO le calcul du régime forcé dû à ces sources indépendantes. Par ailleurs la pulsation mécanique $\Omega$ étant une variable dont dépendent les coefficients de couplage résistif nous avons dû introduire un échange d'information entre le programme SACSO et l'équation mécanique. Ainsi cette dernière est intégrée pendant un pas de calcul au moyen d'une méthode du type Euler qui s'écrit, dans l'hypothèse où on néglige le frottement mécanique du rotor de moment d'inertie $J$ :

$$
\Omega\left(t_{n}\right)=\Omega\left(t_{n-1}\right)+\frac{1}{2 J}\left(\Gamma\left(t_{n}\right)+\Gamma\left(t_{n-1}\right)\right) .
$$


Ainsi le couple $\Gamma$ est calculé au moyen de SACSO grâce à (6) ou (10), et la valeur de $\Omega\left(t_{n}\right)$ trouvée est alors utilisée pour déterminer les couplages dans le pas suivant $\left(t_{n}, t_{n+1}\right)$ de SACSO. La vitesse du rotor ayant une évolution lente ce retard dans le calcul est justifié.

La simulation ainsi mise en œuvre sur calculatrice UNIVAC 1110 demande un temps de calcul de $10 \mathrm{~min}$. et nous fournit les tracés Benson des figures 10 à 12 qui sont à comparer directement aux oscillogrammes des figures 4 à 6 . Ces tracés sont obtenus indifféremment avec un modèle diphasé ou triphasé,

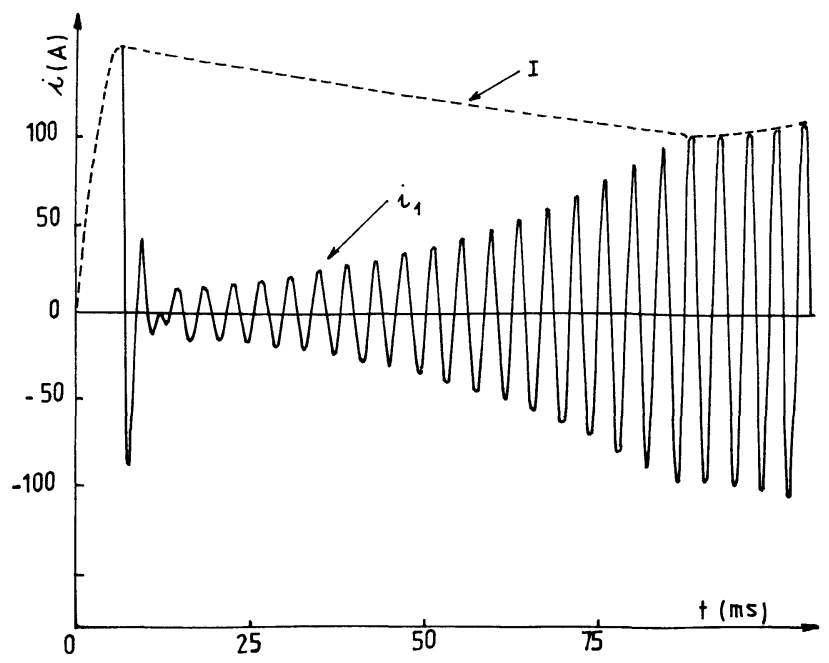

Fig. 10. - Simulation de $i_{1}$ et $I$ au début de la décharge

[Simulation of $i_{1}$ and $I$ at the beginning of the discharge.]

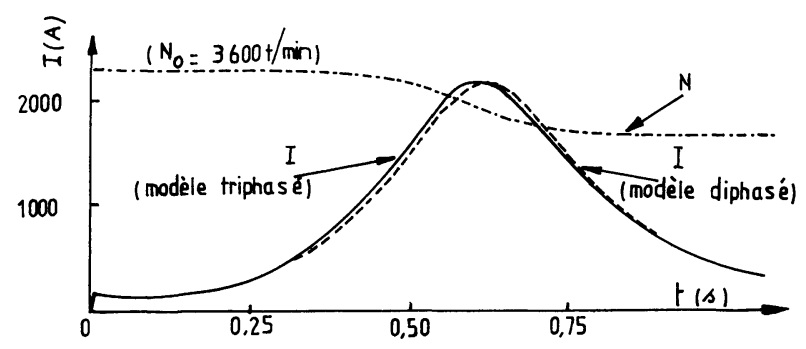

Fig. 11. - Simulation complète de $I$ et $N$ (vitesse du rotor).

[Complete simulation of $I$ and $N$ (speed of the rotor).]

l'accord entre les résultats de ces deux modèles étant très bon.

Le tracé Benson de la figure 10 est à comparer à l'oscillogramme de la figure 4. Dans les deux cas nous trouvons une constante de temps et une fréquence d'oscillation voisines de $37 \mathrm{~ms}$ et $236 \mathrm{~Hz}$.

Quant au tracé Benson de la figure 11 qui donne le courant dans lä charge en fonction du temps, il est à comparer à l'oscillogramme de la figure 5. Ainsi si lors de l'expérience le courant maximal dans la charge est de $2540 \mathrm{~A}$ à l'instant $T=0,7 \mathrm{~s}$ la simulation

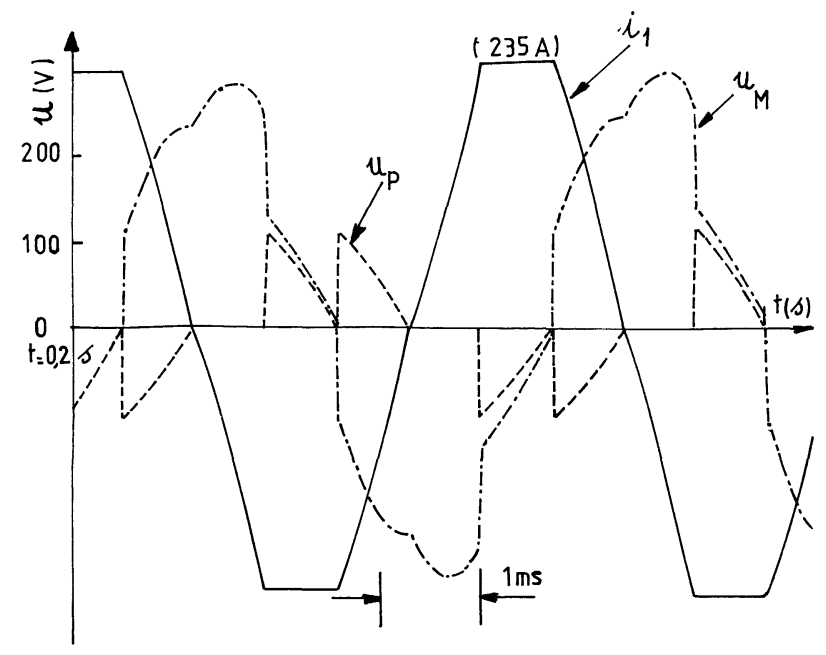

Fig. 12. - Forme des tensions et courants donnés par la simulation lors de la décharge utile.

[Voltages and currents waveforms given by simulation during useful discharge.]

donne une valeur un peu inférieure de $2110 \mathrm{~A}$ à un instant $T=0,64 \mathrm{~s}$.

D'autre part les tracés de la figure 12 donnent les tensions et courants dans la génératrice qui sont comparables à ceux de l'expérience portés sur les oscillogrammes de la figure 6 .

6. Etude directe de «l'amorçage interne ». - Afin de vérifier les résultats de la simulation globale indépendamment de la validité d'un modèle, nous avons effectué [5] au moyen d'une méthode de recherche de valeurs propres, une analyse plus précise de l'amorçage interne de la génératrice asynchrone à vitesse constante. En effet pendant cette période de fonctionnement le pont de Graetz étant en court-circuit et la vitesse du rotor étant pratiquement constante, l'évolution de la génératrice satisfait aux équations linéaires (1) à (5) auxquelles nous devons ajouter :

$$
\begin{aligned}
& i_{\mathrm{d}}=\frac{3}{2} C \frac{\mathrm{d} v_{\mathrm{d}}}{\mathrm{d} t}, \\
& i_{\mathrm{q}}=\frac{3}{2} C \frac{\mathrm{d} v_{\mathrm{q}}}{\mathrm{d} t} .
\end{aligned}
$$

En effectuant le changement de variable suivant :

$$
\begin{array}{ll}
i_{+}=i_{\mathrm{d}}+j i_{\mathrm{q}} & i_{-}=i_{\mathrm{d}}-j i_{\mathrm{q}} \\
j_{+}=j_{\mathrm{d}}+j j_{\mathrm{q}} & j_{-}=j_{\mathrm{d}}-j j_{\mathrm{q}} \\
v_{+}=v_{\mathrm{d}}+j v_{\mathrm{q}} & v_{-}=v_{\mathrm{d}}-j v_{\mathrm{q}}
\end{array}
$$

(où $j$ est le nombre imaginaire pur de module unité), le système linéaire précédent se transforme en deux systèmes imaginaires conjugués découplés : 


$$
\frac{\mathrm{d}}{\mathrm{d} t}\left[\begin{array}{c}
v_{+} \\
i_{+} \\
j_{+}
\end{array}\right]=[P]\left[\begin{array}{c}
v_{+} \\
i_{+} \\
j_{+}
\end{array}\right] \quad \text { et } \quad \frac{\mathrm{d}}{\mathrm{d} t}\left[\begin{array}{c}
v_{-} \\
i_{-} \\
j_{-}
\end{array}\right]=[P]^{*}\left[\begin{array}{c}
v_{-} \\
i_{-} \\
j_{-}
\end{array}\right] \text {, }
$$

où

$$
\begin{array}{ccc}
{[E]=\left[\begin{array}{ccc}
C & 0 & 0 \\
0 & \frac{2}{3} L_{\mathrm{s}} & \frac{2}{3} M \\
0 & \frac{2}{3} M & \frac{2}{3} L_{\mathrm{r}}
\end{array}\right] \quad[F]=\left[\begin{array}{ccc}
0 & 1 & 0 \\
-1 & -\frac{2}{3} R_{\mathrm{s}} & 0 \\
0 & -\frac{2}{3} j M q \Omega & -\frac{2}{3}\left(R_{\mathrm{r}}+j L_{\mathrm{r}} q \Omega\right)
\end{array}\right] .}
\end{array}
$$

Ainsi au moyen des conditions initiales suivant immédiatement la décharge préliminaire du condensateur préchargé, l'amorçage interne de la machine asynchrone s'étudie simplement au moyen d'un seul des deux systèmes précédents. L'intégration de ce système est obtenue grâce à une recherche des vecteurs propres et valeurs propres de $[P]$ ce qui conduit, ces dernières étant simples, à une diagonalisation de $[P]$. Les tensions et courants ainsi déterminés [5] pendant l'amorçage interne sont identiques à ceux donnés par la simulation globale obtenue précédemment au moyen du programme SACSO.

Les valeurs propres $\lambda_{i}$ de $[P]$ constituent les modes libres d'oscillation de la génératrice. Au maximum une seule $\lambda=\alpha+j \omega$ de ces valeurs propres a une partie réelle positive et conduit à un amorçage interne. Nous avons groupé sur les figures 13 et 14 les caractéristiques de cette valeur propre en fonction de la valeur $C$ des condensateurs et de la vitesse de rotation $N_{0}$ du rotor. Nous en déduisons pour $C=6600 \mu \mathrm{F}$ une vitesse critique de la génératrice $N_{\mathrm{c}}=1388 \mathrm{t} / \mathrm{min}$. $\mathrm{Au}$-dessous de cette vitesse critique il n'est plus possible d'obtenir un amorçage quelle que soit la valeur des condensateurs. Précisons que cette valeur de la vitesse critique est en accord avec son estimation expérimentale à partir de la mesure du couple, qui est de $1200 \mathrm{t} / \mathrm{min}$.

7. Conclusion. - La simulation globale du système à l'aide d'un programme SACSO modifié conduit à une description numérique de la décharge d'une génératrice asynchrone sans fer dans une bobine bien cohérente avec l'expérience.

Le fonctionnement global comporte trois phases :

- l'amorçage interne

- la décharge externe

- le désamorçage.

L'amorçage interne a été simulé par deux méthodes : un traitement linéaire rigoureux et le calcul numérique. On a pu ainsi vérifier directement la bonne valeur mathématique du traitement numérique sur une partie de la décharge.

En ce qui concerne la comparaison globale expérience-simulation, l'accord a lieu avec des erreurs maximales de l'ordre de $20 \%$.

La difficulté majeure présentée par la simulation numérique de ce système non linéaire réside dans le temps relatif très long du traitement : 200 périodes

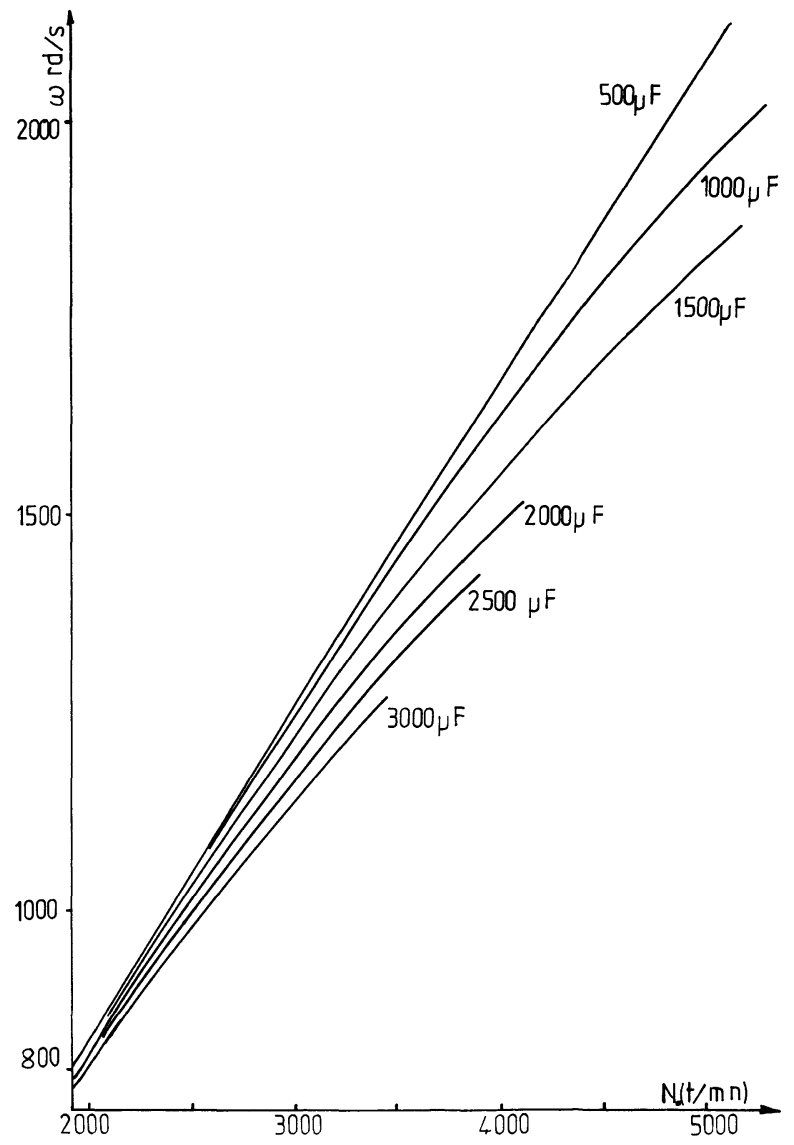

Fig. 13. - Pulsation $\omega$ des tensions et courants lors de l'amorçage interne.

[Pulse frequency $\omega$ during the internal discharge period.]

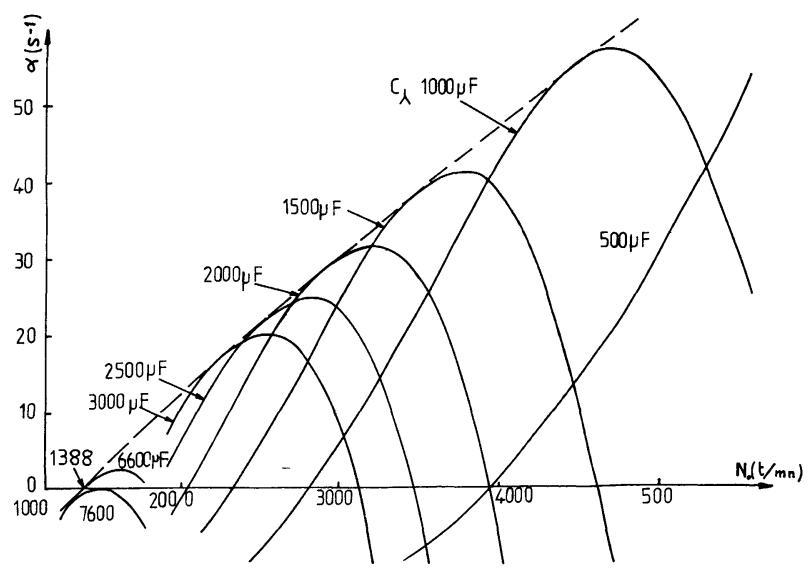

Fig. 14. - Constante de temps $\alpha$ lors de l'amorçage interne.

[Internal discharge time constant $\alpha$.] 
d'un régime oscillant où il faut décrire pour chaque cycle un nombre important de commutations élémentaires dues aux redresseurs. En particulier il faut noter qu'une utilisation du programme SACSO dans laquelle les f.e.m. dynamiques étaient traitées à l'instant $t_{n}$ comme des sources dépendant des courants calculés à l'instant $t_{n-1}$ nous a conduits initialement à des résultats incorrects, très sensibles au pas de calcul. Ce n'est qu'après avoir introduit les couplages résistifs dans SACSO afin d'éliminer ce retard dans le calcul que nous avons obtenu des résultats satisfaisants.

Pour terminer, notons que le modèle ne représente que de manière assez grossière la structure du rotor conducteur massif; l'idée serait de disposer d'une modélisation tridimensionnelle exacte du couplage rotor-machine que nous ne possédons pas. Les écarts expérience-simulation qui subsistent encore sont probablement dus pour l'essentiel à cette question que nous abordons actuellement [8].

Remerciements. - Nous remercions MM. Foch et Davat du Laboratoire d'Electrotechnique et d'Electronique Industrielle de l'ENSEEIH de Toulouse pour leurs conseils lors de la mise en œuvre du programme SACSO.

\section{Bibliographie}

[1] Rioux, C., Thèse de Docteur ès Sciences, Paris (1964).

[2] Rioux, C., Sultanem, F., A solid rotor iron-free asynchronous machine. J. Appl. Phys. 50 (1979) 574.

[3] Rioux, C., Théorie comparative des machines électriques établie à partir des expressions du champ électromagnétique $R G E$, 79 (1970) 415.
[4] Ly, K., Quichaud, G., Sultanem, F., Colloque sur les régimes transitoires des machines et de leurs alimentations, Nancy (1978).

[5] Ly, K., Thèse de Docteur-Ingénieur, Paris (1979).

[6] Schonek, J., Thèse de Docteur-Ingénieur, Toulouse (1977).

[7] Davat, B., Thèse de Docteur-Ingénieur, Toulouse (1979).

[8] Tchakala, E., Rapport de D.E.A. d'Electrotechnique, Paris (1979). 